\title{
Sustainable Supplier Selection Based on Multi- Objective Water Cycle Algorithm
}

\author{
S. Sharanya ${ }^{1}$, C. Veeramani ${ }^{2 *}$ \\ \{ssharanya015@gmail.com ${ }^{1}$, veerasworld@yahoo.com² $\}$ \\ ${ }^{1}$ Research Scholar, Department of Applied Sciences, PSG College of Technology, Coimbatore, India \\ 641004, ${ }^{2}$ Assistant Professor, Department of Applied Sciences PSG College of Technology
}

Coimbatore, India 641004

\begin{abstract}
Evolutionary algorithms (EA) are well-suited for optimization problems with numerous objectives. The Multi-Objective Water Cycle Algorithm (MOWCA) is a comparatively recent approach for determining the Pareto-optimal set for multi-objective optimization problems, and the Sustainable Supplier Selection Problem (SSS) plays an essential part in the optimization of SSS problem. The purpose of this study is to examine the Pareto solutions to the SSS issue using the Multi-Objective Water Cycle Algorithm. Initially, a Multi-Objective Water Cycle Algorithm (MOWCA) encouraged by the water cycle process is presented. The mathematical programming for the SSS problem is then developed. The stated Multi-Objective Programming Problem (MOPP) is then analyzed using the MOWCA to get the pareto solutions. The suggested approach's efficiency is examined utilizing performance metrics such as Generational Distance, Reverse Generational Distance, Delta Metric, and Metric of Spacing. Finally, a comparison study with various recent metaheuristic methods is performed.
\end{abstract}

Keywords: Multi-objective optimization, Water cycle algorithm, Sustainable Problem.

\section{Introduction}

During the last few decades, the SSS problem has received much interest in business literature and practices. Through integrated planning and control outcomes, supply chain management improves a company's industry profitability and customer happiness. Furthermore, as the problems of worldwide increase, the focus of the supply chain is on decreasing operating costs and improving total profitability. As a result, selecting a SSS is a multi objective decision problem. Various methods have been used to identify suppliers and make better decisions. Weber, C. A., et al [1] analysed and categorized the vendor selection process in 74 relevant publications published since 1966.

Most academics examine minimising overall cost, increasing quality, and other factors in the SSSproblems. As a result, this is one of the issues in multi-objective optimization. In Multi-Objective Optimization (MOO), objectives compete with one another. As a result, evolutionary algorithms are being used to tackle Multi-Objective Optimization Problems (MOOP). ChiragBabbar et al.[2]devised a novel mathematical method for choosing a group of vendors and distributing order quantities.To establish some amount of uniformity across diverse targets, Jadidi O. et al [3] devised a normalised goal programmingtechnique. He views the SSS as a MOOP in which the three objectives are to minimise price, rejections, and lead 
time.Their algorithm attempts to select the best vendors, classify them to stores, and determine the optimum values for inventory-related choices. Under the conditions of stochastic demand,Zhiying Liao et al.[5]employed a genetic algorithm to build and solve a MOSS model. SSS considers overall cost, excellence rejection rate, late distribution rate, flexibility rate, as well as demand satisfaction \& capacity constraints.

Yeh W. et al. [6] explored two forms of Multi Objective Genetic Algorithms for resolving the supply chain SSS problem.Multi-product SSS problem with stochastic demand problem was developed P.C. Yang et al. [7], which was solved by using GA. Jain V. et al [8] suggested a two-phase Chaotic Bee Colony algorithm for vendor selection, as well as comparison research on the use of $\mathrm{GA}, \mathrm{ABC}$, and $\mathrm{CBC}$ in resolving the order allocation and SSSproblem. Bakeshlou E. A. et al. [9] build a MOFLPP for a Green SSS with 17 criteria separated into five goals and solve it with a Hybrid Fuzzy Multi Objective Decision-Making algorithm (HMODM).

SSS necessitates the examination of supplier performance under a variety of conditions. These conditions are linked in a variety of ways. Furthermore, it is difficult to get specific preferences of Decision Makers (DMs) since (I) the data is ambiguous, and (ii) improving sustainability is dependent on environmental, social, and economic factors. DMs may be unable to assess suppliers in all of these areas. While one Decision Maker may be an expert in economics, another may be an expert on environmental issues.Foerstl, K. [10] investigated how outstanding Purchasing and Supply Management (PSM) departments detect, analyse, and manage supplier sustainability risks, as well as how sustainability risk management is integrated into supplier management procedures. Jauhar, S.K [11]developed a unique technique that uses DEA to choose the most effective sustainable vendors that meet the most of the established sustainable conditions. Zhou X., [12]developed a unique criterion method for evaluating sustainable vendors based on 3 elements and 6 dimensions, and then introduced acombined evaluation framework based on a novel hybrid info aggregation. A genuine instance of a major supermarket is provided and studied to validate the applicability and efficacy of their suggested technique.

We concentrated on the efficiency of the MOWCA in this study by addressing the supply chain system. There are three objectives to be consider in this SSSproblem. They are, (1) Minimization of the product price, (2) maximization of product quality, (3) maximization of Service quality. The Multi-objective problems are obtaine the pareto optimum solutions via to solving the evolutionary algorithms. Hence, the constructed SSSmodel is solved using four popular population based heuristic methods, which are Multi Objective Genetic Algorithm (MOGA), Multi objective Evolutionary Algorithm using Decomposition (MOEA-D), Multi objective Water Cycle Algorithm (MOWCA) and Multi Objective Dragon-fly Algorithm (MODA),

The prospect of this study states as follows:Section 1 discusses about the pareto solutions for the optimization problem by various types of approaches. Section 2 elaborately discusses multi-Objective Optimization and the MOWCA. Section 3 formulate the problem and section 4 discusses the computational results for the SSS problem. The conclusion is presented in Section 5. 


\section{Multi objective optimization and algorithm:}

The MOOP problem with $\mathrm{k}$ objectives can be expressed as follows:

$\operatorname{Max} Z_{m}(\mathrm{y})$,

subject to

$\mathrm{Ay} \leq,=, \geq \mathrm{b}, \mathrm{y} \geq 0$

where $Z_{m}(\mathrm{y}), \mathrm{m}=1,2, \ldots$, kare an objective function. The set $\mathrm{S}$ contains all MOLPP solutions that are feasible, i.e.

$\mathrm{S}=\mathrm{y} \in R^{n}, \mathrm{Ay} \leq,=, \geq \mathrm{b}, \mathrm{y}>0$.

Multi-Objective Water Cycle Algorithm:

The MOWCA method is described for determining the MOLPP'spareto optimum solution set. This is a new optimization technique for finding a better optimal solution, the WCA has been improved by Alisadolah. He also introduced MOWCA, a tool for tackling multiobjective problems. The MOWCA's fundamental aim is extracted from natural system and the water cycle method. The WCA method is primarily concerned with how streams \& rivers flow downward towards the sea in the actual world. The MOWCA begins with sporadic rain. Before we can define pareto optimum solutions in MOWCA, we must first discover dominance and non-dominance solutions. After discovering the dominant and non-dominant solutions, select the most efficient solutions using the crowding distance mechanism, in the population as sea and river. The choosing of sea and river from the dominated and nondominated solutions impacts the convergence of the proposed method. As a result, the crowding distance is determined for non-dominated solutions. Following that, the sea and river are chosen for the resulting non-dominated solutions.

Next, addition of the number of rivers with a single sea is considered as $\mathrm{P}_{\mathrm{sr}}$. After that, the nominatedstreams chosen for each river and sea are selected by an intensity flow. That is, the selected streams flow directly to the rivers or the sea. if a stream's solution, on the other hand, is superior to that of its connected river, the stream's and river's locations are switched. Similar to this for both the river and the sea. After this, the evaporation will be performed. The raining process is performed, if the evaporation process is satisfied. Else, $d_{\max }$ value will be diminished. Later, the new streams are generated in different positions. This process will continue until the solution is resolved. Finally, choose the sea and rivers for following iteration according to the strategy. Hereby, the MOWCA tends to an unintended move to the best solutions. The detail process of MOWCA is given in the following pesudo code of Algorithm 1. 


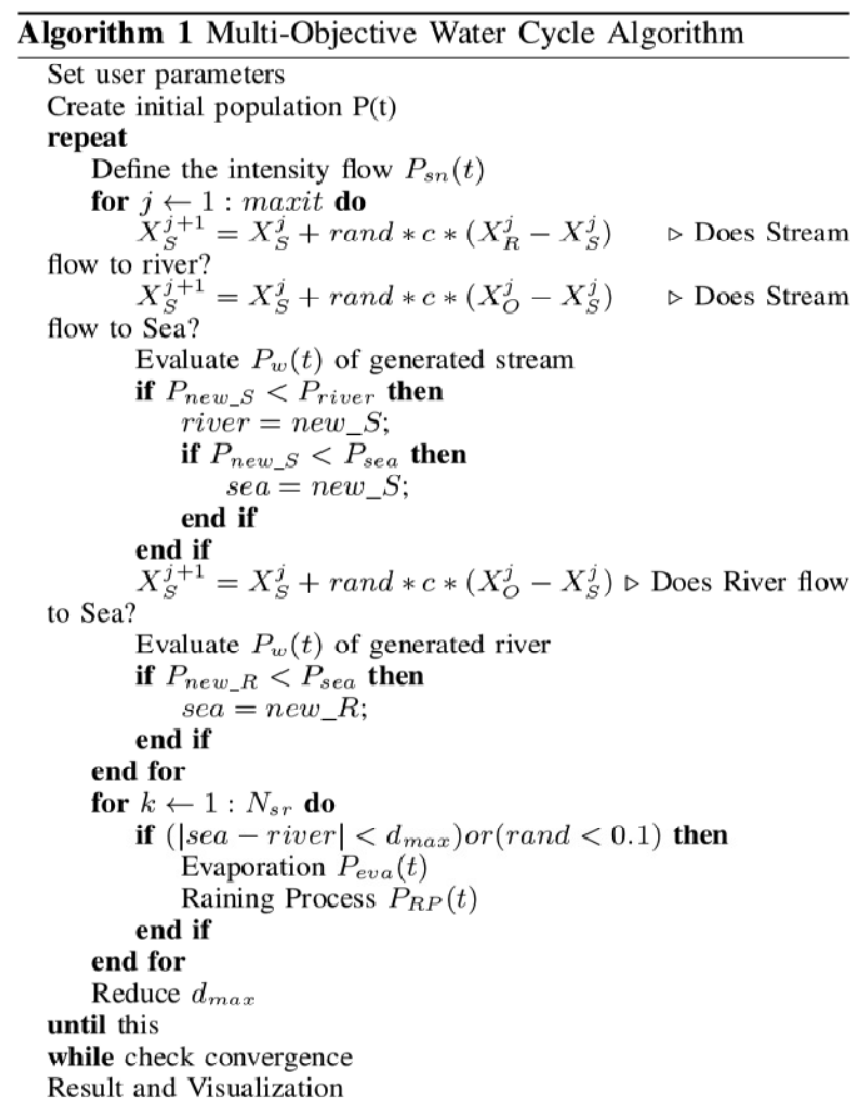

Once the group of paretoresults is available, the efficiency of the proposed algorithm is determined using this pareto archives. Performance metrics [13] are evaluated by MOWCA's overall performance by using the pareto archive. The performance metrics are Generational distance metric (GD), The Reverse Generational Distance (RGD), Metric of spacing (S), Delta Metric (\$Delta\$). We evaluate the performance of the MOWCA based on these performance metrics, and the results are compared to the recent evolutionary methods.

\section{Definition And Formulation Of The Problem}

In this work, we consider the pulp and paper industry sustainable problem [14]. Paper and cellulose-based fiber products are produced in large quantities by the pulp and paper industry. Copy paper, Bulletin papers, manykinds of tissue, cigarette paper, bottle adhesive labels and coffee filters are just a few examples of the items we use on a daily basis. There is an enormous procedure involved in the chain behind these yields. (1) Wood preparation (2) Pulping (3) Bleaching (4) Chemical recovery (5) Pulp drying (non-integrated mills only) and (6) Paper manufacturing, are the six key process areas in the manufacture of paper. 
We propose an assuming paper company in India (X Company) and derive the SSS problem based on the lead time, eminence, pricing, service quality, and $\mathrm{CO}_{2}$ emissions of the supplied products. The manufacturer needs to know efficiency of 18 suppliers with the above different properties. That is, a supplier cannot provide a better quality or service of that product at a lower cost than other competitors. It is clear that there may be some changes in the characteristics of product offered by that supplier. Data for Service quality elements are derived from the idea of Service quality dimension, which is based on 12 questionnaires, including 27 questions provided by[15]. The required information about suppliers are shown in the table 1 .

\begin{tabular}{|c|c|c|c|c|c|}
\hline Suppliers & Price (P) & $\begin{array}{c}\text { Quality (Q) } \\
\%\end{array}$ & $\begin{array}{c}\text { Service } \\
\text { Quality (SQ) }\end{array}$ & $\begin{array}{c}\text { Lead Time } \\
(\mathrm{L})(\mathrm{Days})\end{array}$ & $\begin{array}{c}\mathrm{CO}_{2} \text { Emission } \\
(\mathrm{CE})\end{array}$ \\
\hline 1 & 187 & 75 & 84 & 3 & 40 \\
\hline 2 & 195 & 77 & 76 & 2 & 30 \\
\hline 3 & 272 & 85 & 27 & 4 & 25 \\
\hline 4 & 236 & 86 & 110 & 5 & 22 \\
\hline 5 & 287 & 74 & 94 & 3 & 38 \\
\hline 6 & 242 & 62 & 102 & 6 & 10 \\
\hline 7 & 168 & 73 & 82 & 3 & 24 \\
\hline 8 & 396 & 92 & 63 & 4 & 38 \\
\hline 9 & 144 & 77 & 55 & 2 & 26 \\
\hline 10 & 137 & 69 & 61 & 3 & 18 \\
\hline 11 & 142 & 54 & 122 & 6 & 24 \\
\hline 12 & 196 & 57 & 75 & 5 & 30 \\
\hline 13 & 247 & 77 & 80 & 1 & 55 \\
\hline 14 & 148 & 61 & 121 & 3 & 39 \\
\hline 15 & 294 & 69 & 125 & 4 & 8 \\
\hline 16 & 249 & 94 & 76 & 6 & 6 \\
\hline 17 & 121 & 88 & 114 & 2 & 55 \\
\hline 18 & 269 & 78 & 65 & 1 & 48 \\
\hline
\end{tabular}

Table 1: Data for numerical problem

The mathematical formulation of this SSSproblem is to minimizing the product price, maximizing service quality and the maximizing quality of the product with some constraints. The lead time of the ordered product is considered as 8 days and also number of suppliers in this problem is as 18 to be assumed. The comprehensive formulation is:

subject to

$$
\operatorname{Min} \mathrm{Z}=\left(\mathrm{Z}_{1}, \mathrm{Z}_{2}, \mathrm{Z}_{3}\right)
$$

$$
\begin{aligned}
& \qquad \sum_{\mathrm{i}=1}^{\mathrm{N}} \mathrm{L}_{\mathrm{i}} \mathrm{y}_{\mathrm{i}} \leq 8, \\
& \sum_{\mathrm{i}=1}^{\mathrm{N}} \mathrm{CE}_{\mathrm{i}} \mathrm{y}_{\mathrm{i}} \leq 300, \mathrm{y}_{\mathrm{i}} \geq 0, \mathrm{~N}=1,2, \ldots, 18 . \\
& \text { where } \\
& Z_{1}=\sum_{\mathrm{i}=1}^{\mathrm{N}} \mathrm{P}_{\mathrm{i}} \mathrm{y}_{\mathrm{i}}, Z_{2}=-\left(\sum_{\mathrm{i}=1}^{\mathrm{N}} \mathrm{Q}_{\mathrm{i}} \mathrm{y}_{\mathrm{i}}\right), Z_{3}=-\left(\sum_{\mathrm{i}=1}^{\mathrm{N}} \mathrm{SQ}_{\mathrm{i}} \mathrm{y}_{\mathrm{i}}\right) \\
& \text { Where } \mathrm{y}_{\mathrm{i}} \text { is the product which is supplied by thei }{ }^{\text {th }} \text { supplier, } \mathrm{P}_{\mathrm{i}} \text { is the price of the Product } \\
& \text { supplied by } \mathrm{i}^{\text {th }} \text { supplier, } \mathrm{Q}_{\mathrm{i}} \text { is the quality of the product supplied by the } \mathrm{i}^{\text {th }} \text { supplier, } \mathrm{SQ} \mathrm{Q}_{\mathrm{i}} \text { is the }
\end{aligned}
$$


service quality of the $\mathrm{i}^{\text {th }}$ supplier, $L_{\mathrm{i}}$ is the lead time of the ordering product of $\mathrm{i}^{\text {th }}$ supplier and $C E_{\mathrm{i}}$ is the $\mathrm{CO}_{2}$ emission of the Product supplied by the $\mathrm{i}^{\text {th }}$ supplier.

In general, multiple objectives are in conflict with other objectives. Hence, instead of optimal solution, non-dominant solutions are used. Therefore, multi objective models are used to create a set of different non-dominant solution for the problem. For that reason, the SSS problem is resolved by using the MOWCA technique.

\section{Computational Results}

MOWCA investigates the sustainable MOLPP problem in this section. When utilising an evolutionary algorithm to solve a multi-objective problem, consider the following main concerns to achieve efficiency.

- Maximizing the solution of the spread metric is discovered, allowing as to make the distribution of solution vectors as consistent and dense as possible.

- $\quad$ Reduce the gap between proposed algorithms Pareto front and ideal Pareto front as much as possible.

- The number of items in the Pareto optimum set that were discovered to be maximised.

The MOWCA algorithm is compared to three distinct approaches in this study: MOGA, MOEA-D, and MODA. The MOWCA method was programmed in the MATLAB which consist of 4GB RAM. The application problem is performedby number of independent runs. In this case, Algorithm focuses on MOWCA's performance compared to the results of testing the problem.

In the literature, academics have utilised a variety of performance metrics in their optimization approaches. In this case, statistical findings were achieved by employing performance indicators such as Generational Distance (GD), Reverse Generational Distance (RGD), Delta (DEL), Metric Spread (S). The mean values and standard deviation (SD) values were calculated using the above-mentioned performance measures.

\begin{tabular}{|c|c|c|c|c|c|c|c|c|}
\hline & \multicolumn{2}{|c}{ GD } & \multicolumn{2}{c|}{ RGD } & \multicolumn{2}{c|}{ S DEL } \\
\cline { 2 - 9 } & Mean & STD & Mean & STD & Mean & STD & Mean & STD \\
\hline MOGA & 0.0574160 & N/A & 12.1312964 & N/A & 34.8560316 & N/A & 1.1029206 & N/A \\
\hline MOEA-D & 0.1550793 & N/A & 6.8616203 & N/A & 58.0235030 & N/A & 0.9788471 & N/A \\
\hline MODA & 0.4758639 & N/A & 1247.6604331 & N/A & 52.3434903 & N/A & 1.1906755 & N/A \\
\hline MOWCA & 0.0000540 & 0.0022834 & 0.0006869 & 0.0290085 & 0.2186698 & 9.23346 & 0.0001978 & 0.008353 \\
\hline
\end{tabular}

Table 2: Comparison of different algorithms based on the mean and standard deviation (SD) values of performance metrics.

From table 2, it is clear that, when all four types of algorithms are with compared the mean value of performance measures, the MOWCA is better according to GD, RGD, Metric Spread and DEL. That is, the obtained non-dominated solutions by the MOWCA, indicates nearer extreme solutions and uniform distribution of the objective space, respectively. With the above discussion, MOWCA is better than other existing algorithms. That is, the suppliers are evenly distributed in the objective space while using MOWCA instead of the other comparing existing algorithms. And also, the obtained non-dominated solutions with different methods are shown in figure 1. From figure 1, the MOWCA gives more non-dominated solutions by comparing other existing algorithm. 


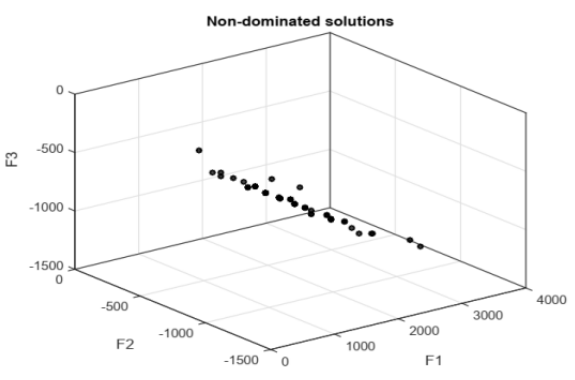

(a)

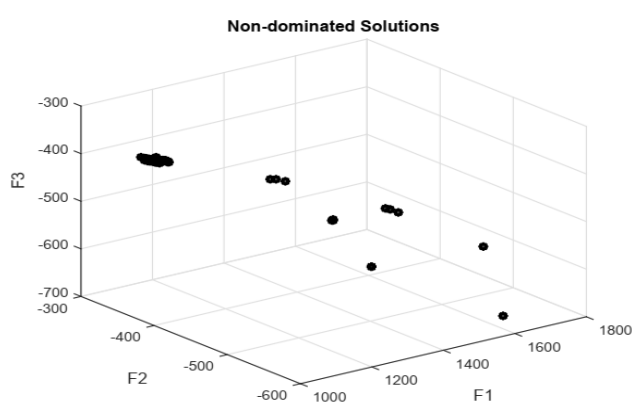

(c)

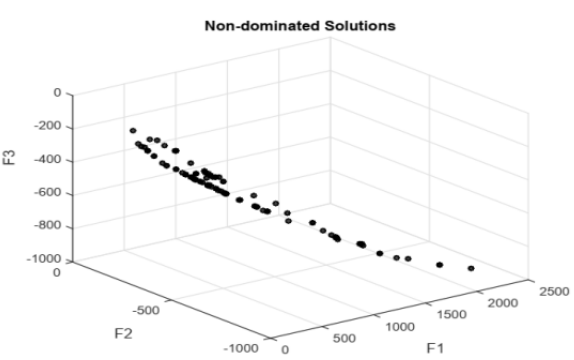

(b)

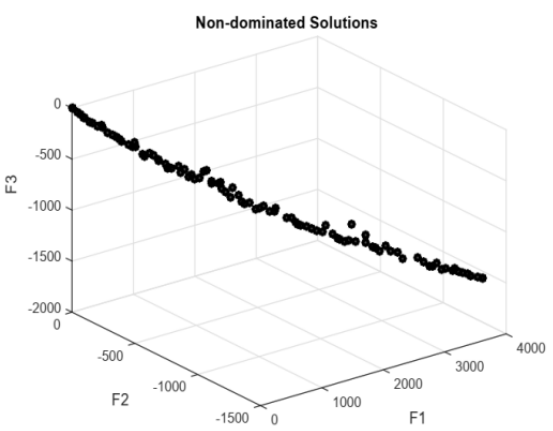

(d)

Figure 1: Distribution of non-dominated solutions and comparisons of the obtained Pareto front with (a) MODA, (b) MOEAD, (c) MOGA, (d) MOWCA for application problem.

\section{Conclusion}

This study introduced the MOWCA, a unique multi-objective heuristic approach for discovering pareto optimum solutions for multi-objective programming problems. The primary goal of this research was to better understand the mechanics of the MOWCA algorithm. The sustainable Multi-Objective Programming Problem has been defined and examined using the MOWCA methodThe statistical findings of the performance measurements show that MOWCA outperforms other methods such as MOGA, MODA, and MOEA-D. The suggested method produces more effective solutions than other algorithms. Furthermore, depending on the difficulty of the optimization problem, MOWCA offers a variety of non-dominant solutions. Future works are being agreed in order to tackle unpredictable and vague real-world difficulties and therefore improve the planned work. 


\section{Acknowledgement}

The authors would like to thank the management and principal, PSG College of Technology for generous support to carry out the research paper.

\section{References}

[1] C. A. Weber, J. R. Current, and W. Benton, "Vendor selection criteriaand methods," Eur.J. Oper. Res., vol. 50, no. 1, pp. 2-18, 1991.

[2] C. Babbar and S. H. Amin, "A multi-objective mathematical modelintegratingenvironmental concerns for supplier selection and orderallocation based on fuzzy QFD inbeverages industry," Expert Syst.Appl., vol. 92, pp. 27-38, 2018.

[3] O. Jadidi, S. Zolfaghari, and S. Cavalieri, "A new normalized goalprogramming model formultiobjective problems: A case of supplierselection and order allocation," Int. J. Prod.Econ., vol. 148, pp. 158-165, 2014.

[4] A. H. Niknamfar and S. T. A. Niaki, "A binary-continuous invasive weedoptimizationalgorithm for a vendor selection problem," KnowledgeBased Syst., vol. 140, pp. 158-172,2018.

[5] Z. Liao and J. Rittscher, "A multi-objective supplier selection modelunder stochasticdemand conditions," Int. J. Prod. Econ., vol. 105, no. 1,pp. 150-159, 2007.

[6] W. C. Yeh and M. C. Chuang, "Using multi-objective genetic algorithmfor partnerselection in green supply chain problems," Expert Syst. Appl.,no. 4, pp. 4244-4253, 2011.

[7] P. C. Yang, H. M. Wee, S. Pai, and Y. F. Tseng, "Solving a stochasticdemand multiproductsupplier selection model with service level andbudget constraints using Genetic Algorithm,"Expert Syst. Appl., vol. 38,no. 12, pp. 14 773-14 777, 2011.

[8] V. Jain, A. Kundu, F. T. Chan, and M. Patel, "A Chaotic Bee Colony approach for supplierselection-order allocation with different discountingpolicies in a coopetitive multiechelonsupply chain," J. Intell. Manuf.,vol. 26, no. 6, pp. 1131-1144, 2015.

[9] E. A. Bakeshlou, A. A. Khamseh, M. A. G. Asl, J. Sadeghi, andM. Abbaszadeh,"Evaluating a green supplier selection problem using ahybrid MODM algorithm," J. Intell.Manuf., vol. 28, no. 4, pp. 913-927,2017.

[10] K. Foerstl, C. Reuter, E. Hartmann, and C. Blome, "Managing suppliersustainabilityrisksin a dynamically changing environment-Sustainablesupplier management in the chemicalindustry," $J$. Purch. Supply Manag.,vol. 16, no. 2, pp. 118-130, 2010.

[11] S. K. Jauhar, M. Pant, and A. Abraham, "Intelligent Data analysis andits Applications,Volume II," vol. 298, pp. 105-117, 2014.

[12] X. Zhou and Z. Xu, "An Integrated Sustainable Supplier SelectionApproach Based onHybrid Information Aggregation,” Sustainability,vol. 10, no. 7, p. 2543, 2018.

[13] C. Veeramani and S. Sharanya, "Analyzing the performance measuresof multi-objective water cycle algorithm for multi-objective linear fractional programming problem," in 2018Second International Conferenceon Intelligent Computing and Control Systems (ICICCS).IEEE, 2018,pp. 297-306.

[14] C. Veeramani and S. Sharanya,An improved Evaporation Rate-Water Cycle Algorithm based Genetic Algorithm for solving generalized ratio problems, Rairo Operations Research 55, S464S480, 2021.

[15] S. M. S. S, D. S. Vijayan, M. Anand, M. Ajona, and T. Jarin, " Biodegradation of P-nitro phenol using a novel bacterium Achromobacterdenitrifacians isolated from industrial effluent water," Water Sci. Technol., vol. 00, no. 0, pp. 1-12, 2021, doi: 10.2166/wst.2021.354

[16] C. Nivetha, D. S. Vijayan, R. Ravishanker et al., Use of Pennywort for nitrogen and phosphate removal from sewage, Materials Today: Proceedings, https://doi.org/10.1016/j.matpr.2020.05.215 
[17] M. Tholkapiyan, A.Mohan, Vijayan.D.S , "A survey of recent studieson chlorophyll variation in Indian coastal waters", IOP Conf. Series: Materials Science and Engineering 993 (2020) 012041, doi:10.1088/1757-899X/993/1/012041.

[18] C Veeramani, M Sumathi, A new method for solving fuzzy linear fractional programming problems, Journal of Intelligent \& Fuzzy Systems 31 (3), 1831-1843, 2016. 